Oscar Aguilar Vidal.

\title{
DON DIEGO BARROS ARANA
}

\section{INTRODUCCFON}

娶

LAMA la atención y es digno de hacer resaltar el caso curioso que ha pasado en nuestra producción intelectual. En ningún país de la América Española se ha desarrollado tanto como en Chile el estudio de las ciencias históricas. Nuestro país puede considerarse como la nación que tiene más completa la historia civil y militar, tal vez a excepción de Méjico.

No hay rincón de la historia-escribe Menéndez y Pelayo-que los chilenos no hayan escudriñado, ni papel de sus archivos y de los nuestros que no impriman e ilustren con útiles comentarios.

Pero ya es hora de que las nuevas generaciones aprovechen este abundante material dejado por nuestros primeros historiadores, haciendo historia crítica y filosófica al estilo de Taine, Buckle y otros, o sintética, psicológica y dramatizada al estilo de Macaulay, Ferrero o Ludwig.

Lastarria, Santa María, Miguel Luis Amunátegui, Isidoro Errázuriz, Vicuña Mackenna y Barros Arana, además de ser la élite de todos los escritores que han estudiado nuestro desenvolvimiento nacional, han trazado al mismo tiempo con su vigorosa acción cívica y cultural las brillantes páginas de nuestra vida republicana.

Lo que caracteriza la grandeza de las obras de la naturaleza $y$ del arte- ha dicho un escritor-es que conservan sus grandes cualidades desde cualquier punto de vista que se presenten delante de nosotros. Sólo es verdaderamente grande y bello lo que no se empequeñece cuando cambiamos nuestra situación al observarlo. 
Son pocos los nombres de los investigadores y sabios contemporáneos chilenos que puedan atravesar triunfantes esa prueba, y entre esos pocos, que en su mayor parte han isido profesores, hay que colocar en primer lugar el nombre del más fecundo y diligente de nuestros historiadores: Diego $\mathrm{Ba}$ rros Arana. La recia personalidad de este hombre se esparce por todo el campo de la vida nacional, ya como hombre de bien, honrado y moral, ya como educador y paladín de las reformas de nuestra enseñanza; ora como humanista, digno sucesor de Andrés Bello, ora como reconstructor de nuestro pasado nacional. Su múltiple actividad se hizo sentir por espacio de medio siglo y su influencia en la vida intelectual chilena fué tan amplia y eficaz que sería injusticia e ignorancia no reconocerla.

Como maestro y rector del Instituto Nacional, le cupo educar con cariño de apóstol a numerosas generaciones de escritores, estadistas y políticos que han figurado o figuran en nuestro reducido mundo intelectual, al mismo tiempo que consagró todas sus energías a la reforma de nuestra instrucción. En estos momentos en que los valores educacionales atraviesan por una dura crisis y en que se desconoce la obra del profesorado nacional, olvidándose de que existieron maestros como los Bello, los Montt, los Amunátegui y los Letelier, todos eminentes hombress en el campo intelectual o político, es necesario levantar muy en alto el nombre de Diego Barros Arana. Han trascurrido más de veinte años de su muerte y aun no se le concede el mármol de la inmortalidad. Es obra de estricta justicia, y muy laudable el esfuerzo que gasta el Gobierno actual, erigirle un monumento al historiador chileno. Pero se honraría dignamente la memoria del egregio ciudadano si se hiciera una obra que compendiara o sintetizara la Historia General de Chile, su obra definitiva.

La Universidad de Concepción, que constituye el núcleo cultural más completo que ha existido en el Sur de Chile, institución donde tienen acogida todas aquellas ideas que vengan a favorecer el desarrollo de nuestra educación, honraría en forma provechosa el centenario del nacimiento de $\mathrm{Ba}$ rros Arana, auspiciando la realización de esta obra que, día a día, se hace más indispensable para nuestra juventud. 


\section{E L HOM B R E}

16 de Agosto de 1830. Aun resuenan los tambores y clarines de los vencedores celebrando el magno acontecimiento político: la llegada de los pelucones al poder. Aun las aristocráticas y apergaminadas calles de la ciudad de Santiago no vuelven a su habitual tranquilidad colonial, cuando en una regia y notable mansión de la calle Ahumada, a pocos pasos de la Plaza de Armas, el júbilo y la alegría han producido tal trastorno en sus moradores que no ha habido tiempo suficiente para preocuparse de los cambios políticos del país.

$\mathrm{Ha}$ sido varón y ha de recibir el mayorazgo de la ilustre casa de los Barros. Don Diego Antonio ya ha designado al rorro la carrera que ha de seguir, de acuerdo con las costumbres de aquellos tiempos; desgraciadamente los años han de derrumbar todas sus esperanzas.

Don Diego Antonio Barros, nacido de respetable familia chilena y distinguido en la política del país, había contraído matrimonio con una hermosa dama argentina, doña Martina Arana y Andonaegui, hermana del Ministro de Relaciones Exteriores del dictador Rosas, don Felipe Arana. Fruto de este matrimonio era don Diego Barros Arana, nacido en tan difíciles momentos, cuando se iniciaba para Chile una nueva era después de los primeros tanteos políticos en materia de gobierno. Dos años más tarde nacía un nuevo hijo, don José Barros. Transcurridos algunos años de lloriqueos y diabluras propias de la edad, los dos muchachos iniciaban sus estudios en el más prestigioso establecimiento de educación de aquel entonces, el Instituto Nacional. Sólo contaba nueve años cuando inició sus estudios preparatorios con mucho latín y teología y casi absolutamente nada de ciencias. Nadie podría haber presagiado el futuro de este muchacho, largo de cuerpo, casi jibado, de aspecto enfermizo, y al que más tarde apodarían sus compañeros con el típico sobrenombre de "palote». Nadie habría creído que este cuerpo débil encerraba un espíritu fuerte y vigoroso para luchar contra las grandes adversidades de la vida. Si bien es cierto que por su tronco ancestral pertenecía a gentes de condiciones especiales de carácter, podría haberse asegurado entonces que llegaría a ser excelente político o profesional, como eran los deseos de su padre. En el hogar, jamás se pudo pensar que toda su dedicación habría de concentrarla en los estudios intelectuales y que haría un sacerdocio de la más noble y desinteresada de las profesiones: la enseñanza pública. 


\section{LOS ESTUDIOS EN EL INSTITUTO NACIONAL}

En 1843, a los trece años, se iniciaba en el curso de humanidades del Instituto, establecimiento donde se acababa de implantar una reforma en el plan de estudios que se ponía en práctica precisamente el mismo año en que Barros Arana entraba a las Humanidades. La reforma consistía en la introducción de algunos estudios elementales de ciencias. Se comprende facilmente que no serían muy vastos los conocimientos que podían adquirirse en este plantel cuando sólo ahora se empezaba por hacer obligatorios estos estudios. Aparte del latín y la filosofía escolástica, que se aprendían con gran extensión, los demás ramos en muy poco estaban considerados.

No le cupo a don Diego recibir conocimientos de Historia de América ni de Chile, ya que estos estudios sólo se implantaron en el establecimiento seis años más tarde en forma muy elemental y llena de errores por el completo abandono en que se hallaba todavía.

Más tarde, arrastrado por la corriente que comenzaba a desarrollarse en la juventud de aquella época, por el gusto a la lectura y los estudios, como consecuencia del movimiento literario del 42, Barros Arana, cuando aun contaba apenas los diez y siete años, comenzó a inclinarse a las lecturas históricas. Leyendo los pocos libros que existían sobre América y Chile, fué despertándose el amor a las ciencias históricas, amor que debía mantener latente hasta los últimos días de su larga existencia.

A los diez y ocho años, siendo estudiante de Leyes, se lanza en sus primeras tentativas de escritor en compañía de su hermano José, que desgraciadamente pronto debía morir. E:tos ensayos juveniles fueron algunas traducciones de novelas francesas de carácter histórico, que publicaron los diarios de aquella época.

Pero he aquí que la fatalidad impide al joven escritor continuar sus estudios de Leyes. En 1849 los médicos recomendaron a la familia retirarlo de la Universidad porque su salud delicada podía hacer peligrar su vida si continuaba forzando su cerebro. Abandona, pues, sus estudios jurídicos y se entrega, en un fundo cercano a la capital, a las faenas agrícolas para reponer su débil cuerpo. Fué mal hacendado; el tiempo que debía emplear en la agricultura lo dedicaba más bien a sus lecturas favoritas. Estudió de preferencia la historia nacional y cuando apenas contaba los veinte años, lanzó al público su primer trabajo original: Estudios históricos sobre Vicente Be- 
navides y las campañas del Sur, estimulado por su buen amigo don Antonio García Reyes quien, además, prologó su obra.

El autor de esta interesante relación-decía García Reyes-ha hecho un buen servicio a nuestra historia. Sin pretensiones muchas veces de una filosofía vana y postiza, se ha contentado con echar las bases sólidas sobre las cuales debe formularse algún día.

Este trabajo, no obstante tener los defectos de un primer ensayo, fué recibido con generales aplausos por la crítica, y se estimul6 al joven escritor a que siguiera cultivando sus aptitudes en los estudios en que se había iniciado. Don Diego Antonio se sintio halagado por este primer triunfo de su hijo y quiso estimularlo al estudio comprándole una riquísima y nutrida biblioteca, en remate público, de mas de trescientos volúmenes sobre historia y geografía americana, muchos de ellos de un alto valor bibliográfico. Esos libros debían ser la base de la biblioteca de nuestro investigador y alcanzarían el día de su muerte a la enorme suma de diez y seis mil volúmenes.

La muerte de su padre, ocurrida en 1851, no interrumpió sino momentáneamente su labor intelectual; entró en seguida en la falange de los jóvenes que ven en la política un campo apropiado para desarrollar su espíritu de lucha. Durante la administración Montt figuró entre la juventud que defendía las libertades conculcadas por el autocratismo, al lado de Amunátegui, Gallo, Matta, Errázuriz y Vicuña Mackenna.

Pacientemente iba nuestro escritor acumulando noticias y documentos guardados por algunas familias; copiaba de las bibliotecas particulares historias más interesantes y escasas, y recibía del extranjero papeles como los enviados desde el Perú por el hijo de O'Higgins, que le servirían como materiales de inestimable valor en la composición de sus historias. Aprovech6 también los interesantes relatos y noticias de numerosas personalidades que habían actuado directamente en nuestra independencia como los generales Prieto, Freire, Las Heras, Pinto y muchos otros.

Al mismo género de estudios se consagraban por aquellos años algunos amigos de colegio de Barros Arana, tales como los hermanos Amunátegui y don Benjamín Vicuña Mackenna, todos ellos considerados como los fundadores de las ciencias históricas en Chile.

Los hermanos Amunátegui, Miguel Luis y Gregorio Víctor, modelos ambos de fraternidady que según un escritor «no vinieron al mundo como los gemelos siameses, en una misma hora, ni unidos como éstos por un nervio simpático que infun- 
día la sangre del uno en las venas del otro; pero cuya existencia moral ha estado desde la cuna de tal modo identificada, que separar sus vidas seria un especie de impiedad, casi un fraticidio", llegaron a ser con Barros Arana sinceros compañeros de labores, produciendo un acercamiento cada vez más estrecho en tal forma que los merecidos triunfos literarios de los hermanos Amunátegui eran celebrados como propios por don Diego.

La amistad literaria con el autor de la Hisloria de Santiago comenz6 en 1855.

La fraternidad literaria que me unía a los Amunátegui se hizo extensiva a Vicuña Mackenna y empeñados todos nosotros en el mismo orden de trabajo, mantuvimos nuestra unión, interesándonos cada cual en la labor de los otros, sin celos de ninguna clase.

El resultado de estas relaciones fué la producción de algunos trabajos bien fundamentados, y tanto los Amunátegui como Vicuña Mackenna y Barros Arana, iniciaron la publicación de obras de prestigio que demostraron el verdadero valor de estos escritores.

La Historia de la Independencia de Chile y Las campañas de Chiloé, dadas a la estampa entre los años 1854 y 1858 , revelan que el escritor ha hecho un sereno y concienzudo estudio de nuestra emancipación política. Es un libro notable el primero por la claridad y el método con que están relacionados los hechos y por la verdad con que están expuestos. Se recomienda - ha dicho Arteaga Alemparte - por el gran caudal de noticias que encierra, recogidas no sólo de los documentos impresos y manuscritos, sino también de boca de los actores que a la sazón sobrevivían al drama de nuestra emancipación. Recomiéndase todavía por el amor a la verdad y el imparcial criterio que han presidido de ordinario a las investigaciones del historiador. En verdad Barros Arana no busca los efectos pintorescos, la rapidez brillante del estilo sino la sinceridad y la perpetuidad de la expresión.

El prestigio de don Diego se iba acrecentando en los centros culturales; prueba de ello es que antes de cumplir los veinticinco años se le honraba nombrándosele miembro Académico de la Facultad de Filosofía y Humanidades de la Universidad. Entrar en ella era ser consagrado como hombre de reconocidos méritos intelectuales y Barros Arana conseguía este triunfo a la edad en que la mayoría de los jóvenes aun no han orientado en definitiva su vida, dominados por los goces propios de la juventud. 
En esta oportunidad iba a ocupar el puesto dejado por el distinguido helenista francés don Luis Antonio Vendel-Heil, y leyó un trabajo sobre la vida y la obra de su antecesor que puede ser considerado como la biografía más correcta desde el punto de vista literario que haya salido de su pluma.

Barros Arana, sin embargo, siguiendo los impulsos de su espiritu liberal se había convertido en un ardiente paladín de estas ideas, atacando al gobierno de los pelucones desde las columnas de $E l$ País y La Actualidad, ambos periódicos de un acentuado tinte reformista. Defensor de las libertades públicas y opositor al exagerado autocratismo del gobierno, no fué sin embargo Barros Arana un publicista de vuelo de la talla de un Lastarria o un Bilbao. Por su carácter y sus condiciones de escritor laborioso y sereno, quizá también por tradiciones de familia, no fué un diarista distinguido. Como diarista-nos cuenta Vicuña Mackenna-era incisivo, franco y enérgico, y en la polémica tan picante y burlón, que no había adversario que no concluyera por cederle el campo. Dividido el partido conservador que había acompañado a Manuel Montt en el gobierno, los ultramontanos formaron alianza con los liberales, mientras los montt-varistas quedaban en el gobierno. Iniciada una ardiente lucha por la prensa y en las reuniones públicas, el gobierno suspende las garantías constitucionales en 1858, cumpliéndose así lo dicho por un escritor argentino:

La única libertad constitucional consagrada en Sud-América es hacerle oposición al gobierno..., cordillera de por medio.

Se acercaba pues para Barros Arana el momento de su ostracismo y estos últimos días de 1858 estarían llenos de dificultades, y muchos sinsabores y amarguras-como consecuencia de las pasiones políticas-perturbarían la tranquilidad personal del escritor. Efectivamente, perseguido por las autoridades subalternas del gobierno, el día 14 de Octubre su hogar era profanado por los esbirros del poder.

Muy de mañana don Diego Barros Arana y don Roberto Souper fueron despertados bruscamente y se les arrancó de sus lechos para ser conducidos a la cárcel; luego se allanaron sus casas, se registraron minuciosamente sus muebles y se desordenó la biblioteca de aquél. El oficial encargado de esta misión, después de un registro prolijo, hizo guardar algunas cartas encontradas en una gaveta y dos pistolas, extraídas de una cómoda, que pertenecían al señor Souper. Como no se encontrara en poder de ellos ni papeles compromitentes, $\mathrm{ni}$ armas, $\mathrm{ni}$ indicios de ninguna especie que pudieran dar motivos para fundar una acusación en forma, después de 12 horas de prisión fueron puestos en libertad. Al día siguiente publicaba su 
Carta Política en la que exponía el atentado violatorio de que había sido víctima por las autoridades administrativas; después de fustigar enérgicamente al gobierno finalizaba: «No terminaré esta exposición sin emitir * una sospecha que se me ocurre y que me hace reír. Mañana los lebreles - de palacio se desatarán para acometerme como lo han hecho otras muchas

- veces. ¡Ladrones! Hacen bien en ladrar los que no tienen otra cosa que \& comer.s

Temiendo por seguridad personal, Barros Arana se vió obligado a ocultarse por algún tiempo y, poco después, a principios de 1859 , a abandonar el país. Su primer viaje al extranjero era consecuencia de un destierro obligado por las circunstancias políticas.

\section{E L OSTRACIS M O}

Desde entonces abandonó por completo la política para entregarse de lleno a sus trabajos de investigación. La política vive de transacciones. Esto la obliga a buscar de preferencia sus favoritos en los términos medios de la inteligencia y del carácter. Nuestro hombre tenía demasiada altivez de espíritu; era incapaz de la lisonja. Se expresaba con una sinceridad chocante para el convencionalismo político, lo que lo alejaba de las situaciones creadas por ese convencionalismo. No estaba destinado a hacer un papel brillante en la política y sólo lo veremos más tarde ocasionalmente actuando muy limitadamente en ella.

Otro era el campo de su enorme actividad.

De Mendoza pasó a Buenos Aires. ¿Qué era lo que atraía a don Diego a la capital del Plata? Un doble motivo de regocijo era para él llegar a la metrópoli argentina: primero, porque en ella encontraría, además de algunos parientes próximos, numerosos amigos como Mitre, Sarmiento y Alberdi, que lo estimaban sinceramente desde su viaje a Chile, y segundo, porque Buenos Aires sería el primer centro de sus investigaciones históricas. Buenos Aires era por aquellos años una ciudad tal vez más pequeña que Santiago. Sufriendo la tiranía de Rosas se encontraba desde 1852 nuevamente en lucha con las provincias.

El encuentro con Mitre le produjo un enorme satisfacción y le fué de gran valor en sus estudios. La amistad con el historiador argentino se remontaba a la época de la primera visita de don Bartolomé Mitre a Chile, allá por el año 1849, amistad sincera y jamás quebrantada. La tiranía de Rosas había obligado a muchos escritores argentinos a abandonar su patria y 
llegados a Chile encontraron la hospitalidad generosa de nuestro país.

Sarmiento, Alberdi, López, como Mitre, vivieron algunos años en el solar santiaguino y no pocas obras inmortales nacieron al calor del terreno ultra-andino.

La amistad de Barros Arana y Mitre debía ser de larga duración y ninguna clase de dificultades la interrumpiría durante toda la vida; juntos en Chile fueron los principales redactores de la prensa liberal y comulgaron ambos las mismas ideas políticas. Combatieron, como pipiolos convencidos, la candidatura de Manuel Montt, lo que obligó al historiador ríoplatense a ausentarse momentáneamente al Perú. Cuando vuelve a Santiago,

Una impresión sentimental muy honda ha de dejar en el corazón de Mitre el conocimiento de una distinguida dama chilena, un ser admirable todo hecho de belleza, de talento y de virtud.

Vuelto Mitre a su patria, debían pasar muchos años para que volvieran a encontrarse estos dos amigos. En Buenos Aires el político argentino puso su biblioteca y todos sus papeles históricos a disposición de don Diego con la más absoluta franqueza.

Las relaciones que habíamos cultivado en Chile en años anteriores se convirtieron entonces en la más estrecha amistad-ha dicho más tarde Barros Arana en su Historia General de Chile-, en una verdadera confraternidad literaria que hemos conservado inalterable a pesar del tiempo, de la distancia y de todas las vicisitudes de la vida, comunicándonos nuestros proyectos literarios y nuestros escritos de cualquier clase que fueran y proporcionándonos recíprocamente, los libros, los documentos y los mapas que podían interesarnos para nuestros trabajos respectivos. Esta amistad de más de cuarenta años, que nada ha perturbado y que nada ha aminorado, amistad sin desconfianzas y sin rivalidades, y en que no han intervenido sino móviles sanos, me ha procurado una no pequeña satisfacción en las aflicciones de la vida y en la carrera de escritor.

No se tienen noticias de su viaje al Uruguay y al Brasil; se sabe, sí, que paś́ a estos países antes de dirigirse a Europa a visitar los museos y bibliotecas de Inglaterra, España y Francia. Llegado en Agosto de 1859 a Londres, don Diego se dedica inmediatamente a trabajar concurriendo al Museo Británico, depósito que guardaba riquezas de un inestimable valor histórico referentes a España y sus colonias. Introducido a ella por la influencia de un antiguo amigo, el general don Juan O'Brien, le cupo trabajar al lado de un conocido escritor so- 
cialista de Francia, Luis Blanc, quien aprovechando su destierro recopilaba en la biblioteca de Londres documentos para la publicación de la Historia de la Revolución Francesa.

En Noviembre, se encontraba Barros Arana en España y en los primeros días de Diciembre de 1859 comienza sus investigaciones en el más rico y considerable depósito de documentos, papeles y libros referentes a la Historia de América: el archivo de Indias de Sevilla. Provisto de un permiso especial de Su Majestad, pudo, por espacio de cuatro meses, examinar todos los papeles que se referían a Chile y algunos relacionados con el Perú y Argentina. En los primeros meses de 1860 trabajaba en el archivo de Simancas y después de aprovecharse de todas las noticias interesantes que encontró en él, pasó a Madrid, donde le fué dado aumentar muy considerablemente la documentación que ya poseía, frecuentando las bibliotecas Nacional de Madrid, la de la Dirección Hidrográfica y muy especialmente la de la Real Academia de la Historia.

Durante su permanencia en España tuvo ocasión don Diego de trabar amistad con distinguidas personalidades hispanas que le facilitaron en muchas ocasiones el desarrollo de su labor. El mismo ha recordado más tarde al historiador don Modesto Lafuente, al literato Juan Eugenio Hartzenbusch, al célebre editor Manuel Rivadeneira, al bibliógrafo don Pascual de Gayangos y a don José Joaquín de Mora, que vivía rodeado de su familia los últimos momentos de su vida y que proporcionó a nuestro investigador numerosas noticias sobre acontecimientos de que había sido testigo o actor en Chile.

A pesar de sus deseos de permanecer más tiempo en España, se vió obligado a trasladarse rápidamente a Francia, donde, según noticias que había recibido, pronto llegaría para establecerse en París, su esposa doña Rosalía Izquierdo.

Por la correspondencia de Barros Arana y Mitre sabemos que el autor de la Independencia de Chile entró en París a dirigir la publicación de una Biblioteca Americana, cuyo objeto principal era dar a la publicidad aquellos manuscritos, documentos o libros impresos que se hubieran hecho raros, aprovechando desde luego Barros Arana el material recogido en los países que había visitado. Al mismo tiempo no descuidaba el examen y revisión de papeles y favorecido por la amistad que lo unía a Claudio Gay, tuvo acceso a la biblioteca donde guardaba una rica colección de piezas referentes a Chile. También se le abren las puertas del archivo particular del general don José de San Martín, rigurosamente guardado por su hija, la señora de Balcarce, que residía en una casa quinta 
de los alrededores de París y que le ofreció a Barros Arana una generosa hospitalidad.

Ese archivo que nadie había consultado hasta entonces con un propósito histórico, contenía los documentos más preciosos, casi todos desconocidos y muchos de ellos de carácter reservado, sobre los complicados acontecimientos en que directa o indirectamente había tenido participación aquel ilustre General. La familia de éste-ha escrito Barros Arana-, que tenía conocimiento de mis escritos históricos, sabía perfectamente que yo había comenzado en ellos la rehabilitación de esa gran figura americana restableciendo la verdad por mezquinas pasiones y por la más negra injusticia, me colmó de atenciones y quiso darme todas las facilidades para adelantar en mis estudios.

Parece que no pensaba regresar a Chile todavía, por los acontecimientos políticos que se estaban desarrollando en este país, y esperaba una mejor oportunidad. En carta dirigida a Mitre, refiriéndose a su patria le dice:

Nada le digo de Chile porque Ud. tendrá noticias más recientes. La pobre patria está sufriendo cuanto es dable. Aquí se encuentran muchísimos proscritos: Santa María, Pedro León Gallo, García Reyes, Guillermo Matta, Guillermo Blest Gana, etc. En el Perú hay más de setecientos hombres conocidos y cerca de doscientos en Mendoza y San Juan.

No teniendo otras actividades que desarrollar en París, don Diego salió a recorrer las bibliotecas de Bélgica, Holanda y Alemania y por el mes de Septiembre piensa ya en el regreso a su patria en vista del cambio político que se ha verificado en Chile.

Ya Ud. tendrá noticias-le dice en una interesante y curiosa carta a su amigo Mitre-que el partido opositor se ha fijado en el general Bulnes como candidato a la presidencia, y que Montt, disimulando tal vez sus propósitos de elevar a Varas, ha hecho proclamar a Pérez, aquel señor conocido con el nombre de Mahoma. Aunque este es un hombre bueno y de alguna inteligencia, su elevación a la presidencia lo pondría en la situación del madero rey de las ranas de la fábula, y Montt sería quien gobernase si se da trazas para mantenerse ligado con aquél. La candidatura de Bulnes, proclamada por la oposición, será quizás el último de los males que Montt infiera a la República, puesto que sólo el deseo de librarse de Varas y de presentar un candidato posible ha hecho que la oposición lo acepte.

Como Ud. sabe los procesos que Montt sabe urdir no han alcanzado hasta mí, y una vez extinguidas las facultades extraordinarias, puedo volver a Chile tranquilamente. Si me es necesario rehacer mi saco de peregrino, volveré a las andadas; es decir, cruzaré la Cordillera y me fijaré en Buenos Aires.

El nuevo gobierno de Pérez, favorable a los liberales, debió apresurar el viaje de nuestro historiador, pues ya en 1860 estaba 
de nuevo entre los suyos después de dos años de peregrinación. Llegado a Santiago comenzó su incansable labor de crítico, bibliógrafo y escritor histórico, publicando en periódicos y revistas de aquel tiempo sus artículos y fundando otros. Fruto del trabajo de este tiempo es una curiosa publicación que llevaba por título Cuadro Histórico de la Administración Montt y que debía aparecer el mismo día que don Manuel Montt depusiera el mando, publicada en colaboración con Santa María, Lastarria y Marcial González.

E1 volumen de seicientas páginas-dice Fuenzalida Grandón en la Vida y Obras de Lastarria -, impreso en una semana, hecho subterráneamente, era anunciado el 18 de Septiembre de 1861 por cartelones fijos en todas las esquinas de la ciudad. El hondo secreto con que había sido hecha la publicación, la curiosidad de saber quién era el autor, la palpitante actualidad del libro, todo contribuyó a un éxito extraordinario, que agotó un grueso tiraje y dejó pingüe ganancia a los autores.

Probablemente no se ha escrito en Chile hasta hoy ningún otro de su clase que le sea comparable, como historia de combate, como ataque sangriento en el fondo, imparcial y sereno en la forma, destinado a provocar un movimiento de reacción en contra de un régimen de gobierno y de los hombres que lo representaron.

\section{EL MAESTRO Y REFORMADOR DE LA ENSEÑANZA}

Hemos llegado a un período brillante en la historia de la instrucción pública en Chile; es el período en que le cabe actuar a don Diego Barros Arana como Rector del Instituto Nacional e iniciar la reforma educacional en este plantel. En 1863, a nombre del Presidente de la República don José Joaquín Pérez, el Ministro de Instrucción lo llamó para que se hiciera cargo de la Rectoría del Instituto.

¿Cómo se desempeñó don Diego en su puesto de educador? No era pequeña la responsabilidad que debía echar sobre sus hombros, pero su versación en materias educacionales eta ya una garantía para salir airoso de todos los obstáculos que se le interpusieran. Aprovechando la experiencia adquirida al visitar los diversos colegios de Europa y América, se dispuso don Diego a introducir trascendentales reformas en el Instituto, que por otra parte había permanecido estagnado y sin progreso alguno desde la reforma de 1843, por la incapacidad de rectores débiles y rutinarios que sólo se preocupaban de sus intereses y tranquilidad personal. Si mala era la instrucción del Instituto, 
se comprende cómo sería ésta en los demás colegios del Estado - particulares. Había, pues, que lanzarse a realizar una amplia reforma que consistiría substancialmente en lo siguiente:

1. Hacer obligatorios en el plan de estudios los principales ramos de las Ciencias experimentales: la Historia Natural, la Química, la Física y las Matemáticas;

2. Ensanchar y mejorar el estudio de las humanidades procurándose buenos textos traducidos o nacionales para esas asignaturas; seleccionar un cuerpo de profesores competentes para la nueva enseñanza;

3. - Reformar los reglamentos y horarios, e impulsar el desarrollo físico y moral de los educandos.

Comenzó por formar un profesorado idóneo y competente en especial en las nuevas asignaturas. No bastaba la erudición de algunos de ellos sino que debían poseer ciertos conocimientos pedagógicos para el mejor resultado de la enseñanza. Suplantó los profesores de curso, de carácter enciclopédico, por los de asignatura. Frecuentó sus clases y él mismo se dedicó a estudiar los nuevos ramos para poder apreciar debidamente los conocimientos que se impartían por los maestros. Los alumnos pudieron ver con asombro al Rector concurrir a todas las clases de humanidades e interrogarlos con tanta competencia como sus propios profesores, lo que le daba un inmenso prestigio sobre el alumnado. Por otra parte, el profesorado cooperó eficientemente a la realización de los propósitos del nuevo rector y varios de esos profesores han llegado a ser más tarde honra del magisterio nacional.

Estableció el sistema de repetidores o maestros-ayudantes, sistema que desgraciadamente más tarde se abandonó. Esto permitía a los jóvenes que tenían la inclinación por la carrera docente, irse iniciando en la práctica de hacer clases, cosa muy digna de aplausos, ya que por aquel entonces no había ningún establecimiento para la formación de los profesores de la enseñanza secundaria; la formación del Pedagógico debía tardar todavía cerca de treinta años.

Para suplir la falta de libros adecuados para los liceos, hizo traducir y adoptar algunos extranjeros y él mismo se dedicó a redactar diversos textos científicos y literarios, algunos de los cuales no obstante haber sido escritos hace más de sesenta años no ha sido posible superarlos y son consultados frecuentemente en muchos colegios de países americanos. Elementos de Geografía Física, primer texto que se conocía en este ramo, fué sin duda una excelente obra para aquellos años y que desgraciadamente hoy resulta atrasada por el enorme progreso 
que han experimentado las ciencias físicas; los diversos textos sobre literatura y muy principalmente el Compendio de Historia de América prueban lo vastos que eran sus conocimientos. El Compendio de Historia de América es, sin exageración alguna, la mejor obra publicada en América sobre esta materia. Era indispensable tener en forma abreviada un cuadro comprensivo y claro de los hechos del descubrimiento, conquista e independencia de todos los países de América, y Barros Arana pudo dar cima en forma acabada a esta obra, gracias a los estudios que desde largo tiempo había estado realizando sobre la civilización del Nuevo Mundo. No es extraño, pues, que algunos países americanos, como Argentina, por ejemplo, hayan adoptado esta obra y hecho varias ediciones de ella.

Bajo su iniciativa se estimuló el amor a la lectura con el fomento de la biblioteca del Instituto Nacional, enriqueciéndola con las obras de los grandes pensadores franceses. El fué quien dió el primer golpe mortal a la enseñanza mecánica, instituyendo en las aulas gabinetes, laboratorios y colecciones de objetos y rebajando los textos al papel subalterno de meros auxiliares del profesor o del alumno. A sus empeños se debe el establecimiento de la enseñanza de la Historia Natural, colocándose al frente de esta cátedra al sabio doctor Philippi, quien publicó también el primer texto de zoología y botánica para la enseñanza secundaria. Merced a estas reformas la enseñanza del Instituto tomó definitivamente rumbo propio, perdió su vestuto carácter clerical y, como era de presumirlo, quedó convertida desde entonces en objeto de hostilidad para los reaccionarios.

La implantación de las nuevas asignaturas en forma obligatoria fué resistida durante algún tiempo, pero Barros Arana no descansó un momento y pudo ver por fin, en 1867, declarada en forma obligatoria la enseñanza de todos los nuevos ramos. Durante sus últimos años se concretó a completar las reformas planteadas en un principio. Como el número de alumnos hubiera aumentado considerablemente, se pensó crear un nuevo colegio similar al Instituto. Don Diego fué de opinión de que al nuevo establecimiento se le cambiara de objetivo y se le diera un cárácter industrial, con lo que ya demostraba poseer un conocimiento exacto de las verdaderas necesidades que tendría nuestro país en el futuro. 
Sin embargo se acercaba la reacción, que había llegado hasta el gobierno y que debería dar un golpe a la enseñanza en la persona del Rector del Instituto.

Desde el advenimiento de don Federico Errázuriz el partido conservador se propuso hacer salir del Instituto al reformador de nuestra educación. En 1872, a raíz de unos desórdenes ocurridos en este colegio se nombró una comisión que informara sobre sus causas. La comisión, en su informe, consideraba que en los últimos sucesos no había podido dejar de influir el no darse a la educación religiosa y a los actos que requiere, toda la importancia, tomando en cuenta la influencia que tiene sobre la conducta de la vida el cultivo y desarrollo de los principios y sentimientos religiosos. Bien se veía el deseo de los miembros de la comisión de lanzar un cargo gratuito a la persona de Barros Arana. Si bien es cierto el Rector era de espíritu liberal, no por eso había dejado de hacer cumplir estrictamente dentro del Instituto los deberes religiosos, que mirados hoy día con imparcialidad y tolerancia más bien constituirían un cargo para el rector por la acentuada tendencia religiosa que dominaba en el colegio.

Fuera de la clase de Religión-ha dicho uno de los alumnos de aquella época, el prestigioso escritor don Gonzalo Bulnes-había asistencia obligatoria a misa los domingos y días festivos. Además anualmente concurríamos en cuaresma a ejercicios que se daban en el mismo establecimiento, y teníamos obligación de confesarnos. La comunión se hacía afuera y corría por cuenta de los padres.

Tomando en cuenta el informe de la comisión, el gobierno dividió las obligaciones del Rector en dos personas y colocó a don Camilo Cobo como principal jefe del establecimiento. Por último, en Marzo de 1873, era destituído don Diego Barros Arana de su puesto por el entonces Ministro de Instrucción Pública don Abdón Cifuentes, de recordada y triste memoria. La instrucción pública sólo le debe a este político el mayor de los retrocesos y el más grande de los desórdenes en el desarrollo de nuestra enseñanza. Para engañar a la opinión y darse aires de liberal, estableció este ministro, bajo el nombre de «libertad de exámenes", una libertad de comercio repugnante, que puso en el mercado público cosas que hasta entonces no se habían podido adquirir sino mediante el estudio y el mérito, los certificado del saber. Felizmente reaccionando pronto ante este estado de cosas, la opinión pública lanzó a la calle al hombre 
iLovePDFhttps://doi.org/10.29393/At68-198OADD10198

Don Diego Barros Arana

401

que no había sabido ni respetar a la enseñanza ni a los hombres más preparados de ella.

Por otra parte los jóvenes estudiantes comprendieron la injusticia que se había cometido, y conservando en sus corazones el cariño por su maestro con la misma firmeza con que en su cerebro guardaron sus enseñanzas, fueron el año de 1875 , en el día de su onomástico el 12 de Noviembre, a hacerle, en número de cerca de dos mil antiguos y actuales alumnos del señor Barros, una manifestación de desagravio y a expresarle que no había sembrado el bien, la virtud y la ciencia en terreno estéril y desagradecido.

(Continuará.) 